\title{
Impact of the strategies implemented by an antimicrobial stewardship program on the antibiotic consumption in the coronavirus disease 2019 (COVID-19) pandemic
}

\author{
Anna Murgadella-Sancho $\mathrm{PhD}^{1}$ (1), Ana Coloma-Conde $\mathrm{MD}^{2}$ and Isabel Oriol-Bermúdez $\mathrm{MD}^{2,3}$ \\ ${ }^{1}$ Pharmacy Department, Moisès Broggi Hospital, Sant Joan Despí, Barcelona, Spain, ${ }^{2}$ Internal Medicine Department, Moisès Broggi Hospital, Sant Joan Despí, \\ Barcelona, Spain and ${ }^{3}$ Clinical Science Department, Faculty of Medicine, University of Barcelona, Barcelona, Spain
}

To the Editor - In December 2019, the first cases of a new coronavirus disease (COVID-19) caused by the severe acute respiratory syndrome coronavirus 2 (SARS-CoV-2) were reported in Wuhan. ${ }^{1}$ This infectious disease spread rapidly and led to the current global pandemic. Even though COVID-19 is a viral disease, the use of antibiotics in these patients has been a common practice, especially at the beginning of the pandemic. In published series of hospitalized patients with COVID19 , the prevalence of antibiotic use ranges from $72 \%$ to $95 \%{ }^{2,3}$ However, in published reviews, the prevalence of bacterial coinfection in these patients is $8 \%$ and that of superinfection is $14.3 \%{ }^{4,5}$ Therefore, the systematic use of antibiotics does not seem to be an adequate strategy and can lead to toxicity and resistance issues. ${ }^{6}$

In our center, Moisès Broggi Hospital (a 380-bed regional hospital, located in Barcelona, that serves an area of 425,000 inhabitants), we conducted a before-and-after study to compare the evolution of antibiotic consumption in conventional hospitalization between 2019 (pre-COVID-19) and 2020 (COVID-19), and to analyze the effect of antimicrobial stewardship (AMS) team strategies applied during the COVID period.

Between March and May 2020 the AMS team was unable to conducte its usual activities, but the following measures were implemented to avoid antibiotic overuse. First, a multidisciplinary work team (intensive care, anesthesia, internal medicine, pharmacy and emergency medicine departments), led by members of the AMS team, was created to draw up a protocol for the management of COVID-19 patients. This document included recommended antibiotic regimens (ceftriaxone and azithromycin) and their duration ( 5 and 3 days, respectively). Second, in the computer system, the duration of these treatments was fixed by default to the days defined in the protocol (with prior notification of this measure to all professionals). Prior to completion, antibiotic treatments were reviewed by the clinical pharmacist of the AMS team. After the first COVID-19 wave, the limited duration of treatments in the computer system and the recommendation to use azithromycin were removed from the protocol.

To analyze the evolution of consumption within the hospital and to be able to compare it with that of other hospitals, we used the consumption of antibiotics calculated as defined daily dose (DDD)/100 bed days as an indicator.

Figure 1 shows the monthly consumption of antibiotics in our center in 2019 and 2020, expressed in DDD/100 bed days. During 2020 antibiotic consumption was lower than in 2019 except for

Author for correspondence: Anna Murgadella-Sancho, E-mail: anna. murgadellasancho@sanitatintegral.org

Cite this article: Murgadella-Sancho A, Coloma-Conde A, and Oriol-Bermúdez I. (2022). Impact of the strategies implemented by an antimicrobial stewardship program on the antibiotic consumption in the coronavirus disease 2019 (COVID-19) pandemic. Infection Control \& Hospital Epidemiology, 43: 1292-1293, https://doi.org/10.1017/ ice. 2021.237
March 2020, when the onset of the pandemic caused a punctual increase of the indicator. The mean global consumption of antibiotics during hospitalization was lower in 2020 than in 2019, with statistically significant differences between both periods (57.8 DDD/ 100 bed days vs $64.7 \mathrm{DDD} / 100$ bed days; $t$ test $P=0.045)$. From March to April 2020, the most used antibiotics were azithromycin (16.1 DDD/100 bed days) and ceftriaxone (5.8 DDD/100 bed days). However, piperacillin-tazobactam ranked first in antibiotic use (5.4 DDD/100 bed days) in May 2020.

Previous studies conducted by Abelenda-Alonso et $\mathrm{al}^{7}$ and Grau et $\mathrm{al}^{8}$ analyzed antibiotic use measured with this indicator during the first wave of the pandemic. Unlike our study, the researchers observed an increase in overall antibiotic use, although our data do not include antibiotic use in critical patients. On the other hand, our study does coincide with the previous studies in the identification of a biphasic pattern of antibiotic use with a first peak, during which a higher antibiotic use was detected due to community infections and, later, a second peak during which the increase in antibiotic use included and increase in broad-spectrum antibiotics.

Our experience shows how the adaptation of AMS to the new healthcare reality through the implementation of macro strategies made it possible to control antibiotic use during the first wave when the healthcare pressure did not allow the activity of the AMS team. After the first pandemic wave, hospital activity began to normalize and allowed the usual individualized AMS interventions, which also made it possible to maintain the levels of antibiotic use below those observed in the previous year.

In conclusion, the in-hospital strategies implemented during 2020 by the AMS team contributed to reduce antibiotic use in noncritical patients despite the COVID-19 pandemic.

Acknowledgments. We want to thank all our colleagues: doctors, pharmacists, nurses, microbiologists, cleaning staff and other hospital staff for their work and involvement in facing the COVID-19 pandemic.

Financial support. No financial support was provided relevant to this article.

Conflicts of interest. All authors report no conflicts of interest relevant to this article.

\section{References}

1. Huang C, Wang Y, Li X, et al. Clinical features of patients infected with 2019 novel coronavirus in Wuhan, China. Lancet 2020;395:497-506.

2. Zhou F, Yu T, Du R, et al. Clinical course and risk factors for mortality of adult inpatients with COVID-19 in Wuhan, China: a retrospective cohort study. Lancet 2020;395:1054-1062.

3. Langford BJ, So M, Raybardhan S, et al. Antibiotic prescribing in patients with COVID-19: rapid review and meta-analysis. Clin Microbiol Infect 2021;27:520-531. 


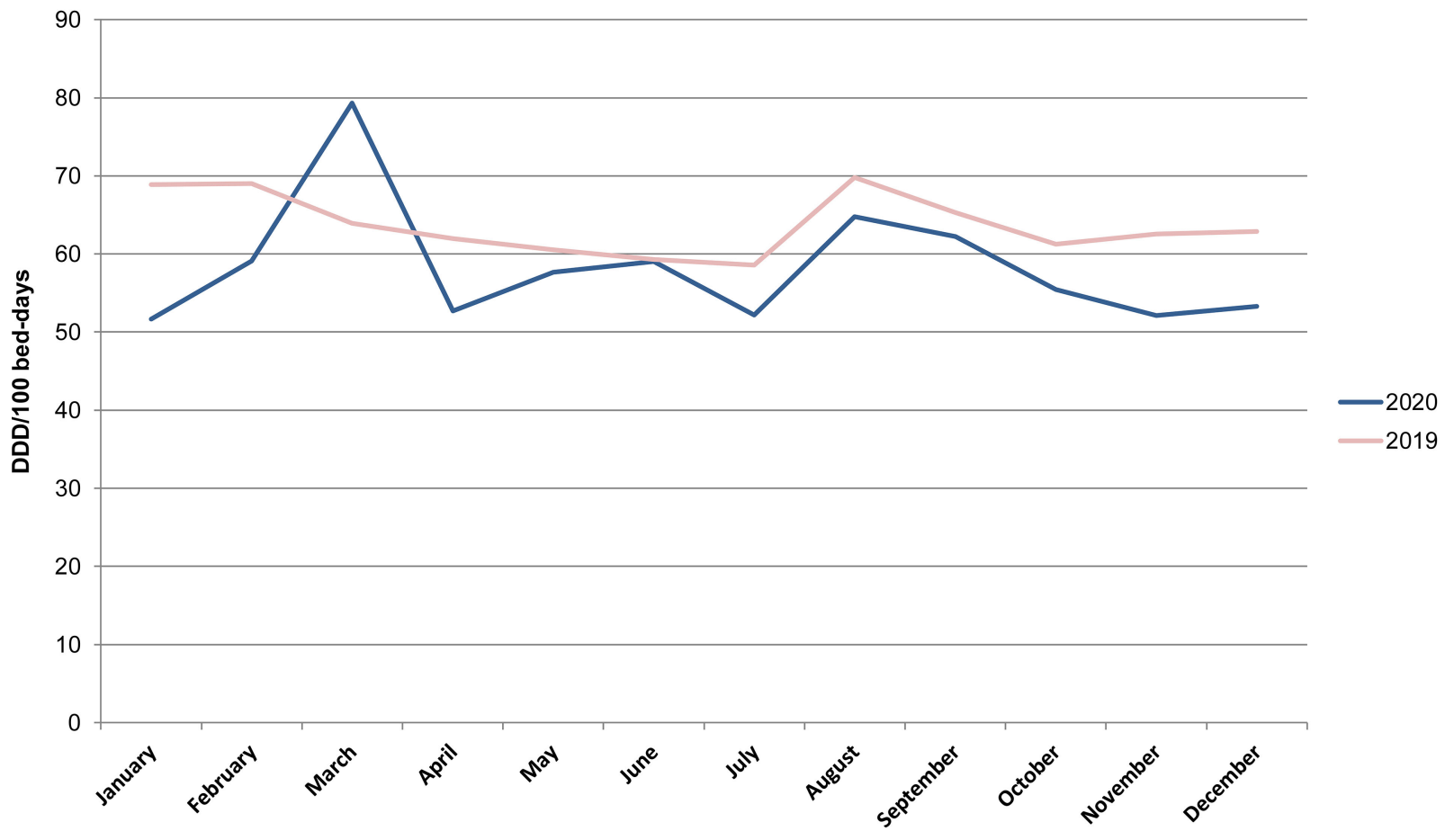

Fig. 1. Global antibiotic consumption.

4. Rawson TM, Moore LSP, Zhu N, et al. Bacterial and fungal coinfection in individuals with coronavirus: a rapid review to support COVID-19 antimicrobial prescribing. Clin Infect Dis 2020;71:2459-2468.

5. Langford BJ, So M, Raybardhan S, et al. Bacterial coinfection and secondary infection in patients with COVID-19: a living rapid review and meta-analysis. Clin Microbiol Infect 2020;26:1622-1629.

6. Rawson TM, Moore LSP, Castro-Sanchez E, et al. COVID-19 and the potential long-term impact on antimicrobial resistance. J Antimicrob Chemother 2020;75:1681-1684.
7. Abelenda-Alonso G, Padullés A, Rombauts A, et al. Antibiotic prescription during the COVID-19 pandemic: a biphasic pattern. Infect Control Hosp Epidemiol 2020;41:1371-1372.

8. Grau S, Echeverria-Esnal D, Gómez-Zorrilla S, et al. Evolution of antimicrobial consumption during the first wave of COVID-19 pandemic. Antibiotics (Basel) 2021;10(2).

\title{
Targeted coronavirus disease 2019 (COVID-19) surveillance for elective procedures: Lessons for resource conservation during a pandemic and beyond
}

\author{
David X. Zheng $\left.\mathrm{BA}^{1}{ }^{(}\right)$, Michelle T. Hecker $\mathrm{MD}^{2}$ and Amy J. Ray MD, $\mathrm{MPH}^{2}$ \\ ${ }^{1}$ Case Western Reserve University School of Medicine, Cleveland, Ohio and ${ }^{2}$ Department of Medicine, The MetroHealth System, Cleveland, Ohio
}

To the Editor-The coronavirus disease 2019 (COVID-19) pandemic has disrupted healthcare delivery worldwide. In mid-March 2020, hospitals across the United States temporarily suspended all elective procedures to mitigate risk of severe acute

Author for correspondence: David X. Zheng, E-mail: dxz281@case.edu

Cite this article: Zheng DX, Hecker MT, and Ray AJ. (2022). Targeted coronavirus disease 2019 (COVID-19) surveillance for elective procedures: Lessons for resource conservation during a pandemic and beyond. Infection Control \& Hospital Epidemiology, 43: 1293-1295, https://doi.org/10.1017/ice.2021.252 respiratory syndrome coronavirus virus 2 (SARS-CoV-2) transmission and to preserve resources to meet clinical demand and ensure workforce preservation (eg, surge planning, employee testing). ${ }^{1,2}$ Although deemed "elective" in that they are nonemergent, elective procedures are essential for patient health and solvency of the nation's healthcare system. ${ }^{3}$

As elective procedures resume, the downstream consequences of their initial delay are becoming apparent. Disruption to patient care may negatively impact outcomes, ${ }^{4}$ and the backlog of elective 